\title{
Large internal dipole moment in InGaN/GaN quantum dots
}

Cite as: Appl. Phys. Lett. 97, 063103 (2010); https://doi.org/10.1063/1.3477952

Submitted: 16 June 2010 • Accepted: 20 July 2010 • Published Online: 09 August 2010

Irina A. Ostapenko, Gerald Hönig, Christian Kindel, et al.

\section{ARTICLES YOU MAY BE INTERESTED IN}

Spectral diffusion time scales in InGaN/GaN quantum dots

Applied Physics Letters 114, 112109 (2019); https://doi.org/10.1063/1.5088205

Multi-excitonic complexes in single InGaN quantum dots

Applied Physics Letters 84, 4023 (2004); https://doi.org/10.1063/1.1751214

Permanent dipole moment and charges in colloidal semiconductor quantum dots

The Journal of Chemical Physics 111, 6955 (1999); https://doi.org/10.1063/1.479988

四 QBLOX

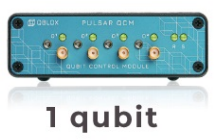

Shorten Setup Time Auto-Calibration More Qubits

Fully-integrated Quantum Control Stacks Ultrastable DC to $18.5 \mathrm{GHz}$ Synchronized $<<1 \mathrm{~ns}$ ultralow noise

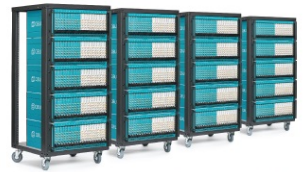

100 s qubits

visit our website > 


\title{
Large internal dipole moment in InGaN/GaN quantum dots
}

\author{
Irina A. Ostapenko, ${ }^{\text {a) }}$ Gerald Hönig, Christian Kindel, Sven Rodt, André Strittmatter, \\ Axel Hoffmann, and Dieter Bimberg \\ Institut für Festkörperphysik, Technische Universität Berlin, Hardenbergstraße 36, 10623 Berlin, Germany
}

(Received 16 June 2010; accepted 20 July 2010; published online 9 August 2010)

\begin{abstract}
Direct observation of large permanent dipole moments of excitonic complexes in InGaN/GaN quantum dots is reported. Characteristic traces of spectral diffusion, observed in cathodoluminescence of InGaN/GaN quantum dots, allow deducing the magnitude of the intrinsic dipole moment. Our experimental results are in good agreement with realistic calculations of quantum dot transition energies for position-dependent external electric fields. (c) 2010 American Institute of Physics. [doi:10.1063/1.3477952]
\end{abstract}

Nitride-based self-assembled semiconductor quantum dots (QDs) have attracted growing interest in recent years for their potential application in optoelectronic devices, such as green and blue light emitting diodes and laser diodes, ${ }^{1}$ and in quantum information processing, like high-temperature single-photon emitters on demand. ${ }^{2,3}$ Wurtzite InGaN and $\mathrm{GaN}$ are remarkable for their strong spontaneous macroscopic polarization. ${ }^{4}$ The built-in piezoelectric and pyroelectric fields ${ }^{5,6}$ tremendously affect optical properties of nitride heterostructures via the Quantum Confined Stark Effect (QCSE). Detailed understanding of the interplay between confined charge carriers and electric fields is crucial for improvement of nitride QD-based devices. Theoretical investigations ${ }^{5-8}$ of InGaN/GaN QDs and a few experimental reports on the QCSE in single InGaN/GaN QDs (Refs. 9 and 10) were reported so far. Experimental evidence of the intrinsic dipole moment is up to now lacking.

The QCSE is suggested to be the cause of spectral diffusion, such as luminescence line broadening, ${ }^{11}$ intensity quenching and random energy shifts of single QD emission lines. ${ }^{12-18}$ One can take advantage of spectral diffusion to assign individual lines to distinct QDs, as reported in microphotoluminescence ( $\mu$ PL) (Refs. 9, 11, and 12) and cathodoluminescence $(\mathrm{CL}){ }^{13,19,20}$

In $\mathrm{CL}$ experiments on single InGaN/GaN QDs, we observe unique spectral diffusion patterns. These patterns cannot be explained within the existing model of charging and discharging of defects in the vicinity of a QD. We propose a novel mechanism, based on the interaction of a QD and a gradually changing field of a propagating charge, and are able to deduce the value of the dipole moment purely from the QD emission energy trace. We substantiate our experimental observations with realistic calculations.

We investigated different samples of InGaN/GaN QDs grown by metal-organic chemical-vapor deposition on $\mathrm{Si}(111)$ substrate [for further details see Ref. 19]. The InGaN layer has a nominal thickness of $2 \mathrm{~nm}$ and is capped with 20 $\mathrm{nm}$ GaN. The samples were covered with metal shadow masks to allow the investigation of individual QDs. ${ }^{20}$ Singledot spectroscopy at liquid helium temperature was performed with a scanning electron microscope JEOL JSM 840 equipped with a CL setup, with a spectral resolution of $310 \mu \mathrm{eV} .^{19}$

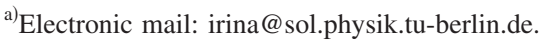

Figure 1 shows an example of the temporal variation in CL spectra for InGaN/GaN QDs with $100 \mathrm{~ms}$ integration time per spectrum. We observe here a typical pattern of emission line oscillations (marked with ellipses in Fig. 1). These oscillations with amplitudes up to $4 \mathrm{meV}$ occur repeatedly for many investigated QDs in different samples, with a random appearance for each QD. We call this pattern " $\chi$ " for simplicity. It appears always in the same manner: the line bends to lower energies, then blinks off and reappears with a blueshift, returning to its mean position afterwards. Such a line trajectory has not been reported yet and does not appear in $\mu \mathrm{PL}$ experiments of the same samples.

Spectral diffusion is commonly explained as follows: free carriers trapped in defects in the vicinity of the QD and in neighboring QDs induce randomly fluctuating local electric fields and thus cause an energy shift of the luminescence lines via the QCSE. ${ }^{9,11,12}$ The dependence of transition energy shift $\Delta E$ on the external electric field $F$ can be expressed as ${ }^{21}$

$$
\Delta E(F)=\sum_{i=x, y, z}\left(\mu_{i} F_{i}-\alpha_{i} F_{i}^{2}\right),
$$

where $\mu_{i}$ are the components of the QD excitonic dipole moment and $\alpha_{i}$ is related to excitonic polarizability.

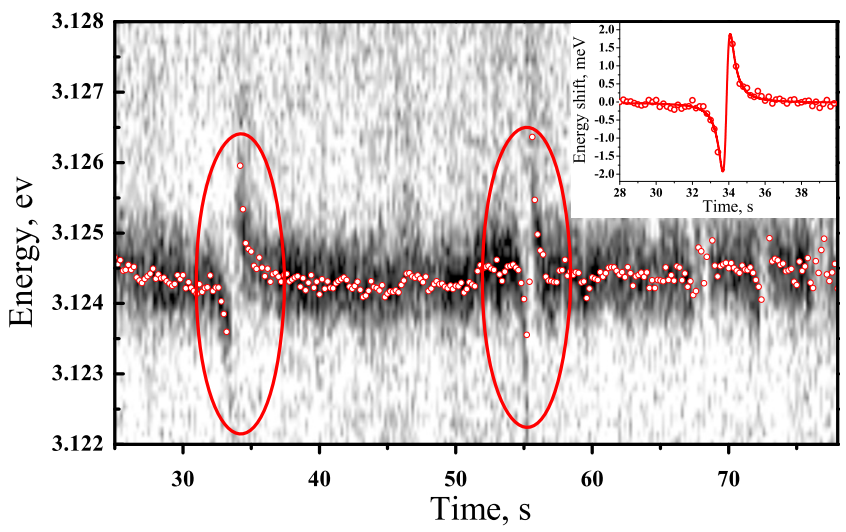

FIG. 1. (Color online) Temporal evolution of CL spectra showing a single emission line from an InGaN/GaN QD. The intensity is coded in gray scale and the integration time for each spectrum is $100 \mathrm{~ms}$. Circles result from Gaussian fits for the peak center energy. Ovals depict typical $\chi$-patterns. One of them is presented in the inset, as time-dependence of energy shift. The solid curve in the inset results from a fit with $\Delta E(t)=\mu_{z} F_{z}(t)$ $\sim\left(t /\left(\text { constant }+t^{2}\right)^{3 / 2}\right)$ [see Eq. (2)]. 
However, the observed $\chi$-pattern cannot be explained with random charging and discharging of defects. Stochastic carrier trap and release processes would result in random discrete energetic jumps or linewidth broadening ${ }^{11}$ and could not give the smooth trajectory shown in Fig. 1, with the same geometry for all occurrences. Many lines show $\chi$-patterns but only a few are synchronous in time. Therefore, the origin must be close to the QD: fluctuating charges on interfaces or in traps lying at larger distances relative to the interdot spacing would result in similar field changes for several neighboring QDs, giving a similar spectral diffusion pattern for all spectral lines.

For the occurrence of the $\chi$-pattern the following conditions must be fulfilled: (1) the field $F$ changes gradually for a continuous, smooth change in peak position. (2) The variation in $F$ accounts for both positive and negative energy shifts of equal magnitude around the mean emission energy. This cannot be fulfilled by a quadratic response of the system to the changes in $F$, and consequently $\mu_{i} F_{i} \gg \alpha_{i} F_{i}^{2}$ in Eq. (1). Hence, the observed patterns present direct experimental evidence of the large dipole moment in InGaN/GaN QDs. (3) For the abrupt change of sign of the line shift, the field $F$ changes its direction relative to the position of the QD once.

For quantitative estimations, we have to apply a detailed model, describing the pattern. The only originator of the electric field fulfilling the above three conditions is a charge, propagating smoothly through the material. We assume a charge $q$ that propagates through the material approximately on a straight line with an average speed $v_{\mu}$, and consider the induced electric field within the QD and the resulting timedependent line shift. More complex charge trajectories do not change the results significantly.

The central symmetry of the $\chi$-pattern with the same amplitude in positive and negative shift direction is only achieved when the propagation direction has a component (anti-)parallel to $\mu$. The field created by a moving charge fulfills all three conditions above. The induced oscillation of the emission energy around its average energetic position $\Delta E[F(t)]$ (see inset in Fig. 1) is

$$
\Delta E[F(t)] \approx \vec{\mu} \cdot \vec{F}=\mu F_{\mu} \sim \mu \frac{t v_{\mu}}{\left(d_{\perp}^{2}+t^{2} v_{\mu}^{2}\right)^{3 / 2}},
$$

with time $t=0$ in the center of the $\chi$-pattern, $v_{\mu}$-average charge velocity along growth direction, $d_{\perp}$-lateral distance between the charge and QD center at $t=0$.

Calculations for InGaN/GaN QDs deliver field strengths in the dot center up to $1.6 \mathrm{MV} / \mathrm{cm}$ (Refs. 5 and 8) and a significant permanent dipole moment due to the separation of electron and hole states within a QD. ${ }^{6}$ Here we theoretically investigate the excitonic dipole moment and the influence of additional electric fields and nearby charges on the exciton emission energy.

We performed self-consistent Hartree calculations for an exciton trapped in lens shaped QDs. The calculations, based on eight-band $\mathbf{k} \cdot \mathbf{p}$ states, include strain, piezoelectricity and pyroelectricity, spin-orbit, and crystal-field splitting. The lateral diameter of the model QD presented here is $5.2 \mathrm{~nm}$ and its height $2 \mathrm{~nm}$. The QD is placed in a $2 \mathrm{~nm}$ thick $\operatorname{In}_{0.1} \mathrm{Ga}_{0.9} \mathrm{~N}$ layer, with the In content inside the QD linearly rising up to a maximum of $\operatorname{In}_{0.49} \mathrm{Ga}_{0.51} \mathrm{~N}$ at the center of the QD. This model system is embedded in a matrix of pure GaN. The parameters were chosen to match the structural investiga-

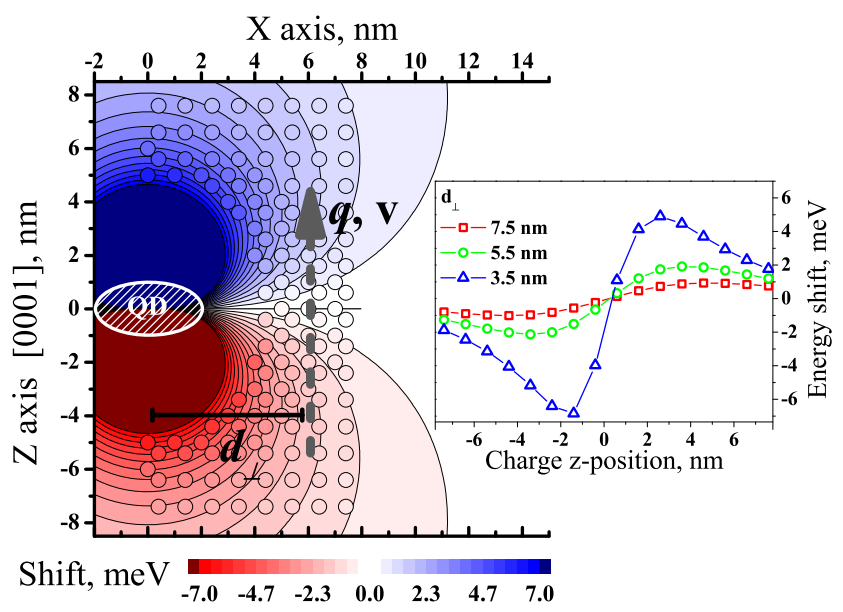

FIG. 2. (Color online) Contour plot of the calculated exciton energy shift for a point charge at $(x, z)$. The QD center is located at $x=0, z=0$. The circles represent results of Hartree calculations for each charge position. The color-coded contour plot results from a fit $\Delta E[\vec{F}(x, z)]=\mu_{z} F_{z}+\alpha F^{2}$. Inset shows the energy shift of an emission line of the model QD for a charge of different lateral distances, $d_{\perp}$, to the QD.

tions of the samples. ${ }^{19}$ The predicted exciton emission energy is $\sim 2.92 \mathrm{eV}$. The dipole moment, calculated from the electron and hole functions density distribution, is $\mu_{z}=1.9$ $\times 10^{-28} \mathrm{C} \cdot \mathrm{m}=1.2 e \cdot \mathrm{nm}$ and $\mu_{x, y}=0$. Change in the model QD structure, leading to the change in the emission energy within the experimentally observed range, does not result in significant change in $\mu$. Applying homogeneous capacitorlike fields in $x, y$ and $z$ direction with a magnitude of $\sim 10 \mathrm{kV} / \mathrm{cm}$, chosen to reproduce the observed energy shifts, yields the same $\mu$.

It is, however, not obvious, to what extent Eq. (1) is applicable for electric fields, that are spatially inhomogeneous across the QD. To verify our model of a traveling charge, we also calculated the exciton energy shifts, caused by external electrostatic fields of a negative elementary point charge, placed onto different positions close to the model QD-see Fig. 2. The circles depict numerical results, the contour plot shows a fit with Eq. (1), resulting again in the same $\mu$. The inset of Fig. 2 shows line traces for different constant lateral distances to the QD. It is easy to see the similarity to the observed experimental data (inset Fig. 1). We find the energy shifts, induced by nearby point charges, still well described by Eq. (1). Particularly our simulations show that the $\chi$-patterns are induced by external fields, more than two orders of magnitude smaller than the built-in fields of the QDs. Hence, the energy shift, caused by the quadratic term in Eq. (1), is much smaller than the one caused by the linear term, and it is justified to set $\alpha_{x, y, z}=0$ in the analysis of the experimental results.

We can now directly deduce the value of the excitonic dipole moment from the amplitude of the $\chi$-pattern. The average QD radius and average distance between the QDs is $2.5 \mathrm{~nm}$ and $10 \mathrm{~nm}$, respectively. ${ }^{19}$ Then with the $d_{\perp}$ in the range of 2.5 to $8 \mathrm{~nm}$ and a maximum observed shift amplitude of $4 \mathrm{meV}$, we obtain dipole moment values between $\mu_{z}=0.7 \times 10^{-28} \mathrm{C} \cdot \mathrm{m}=0.3 e \cdot \mathrm{nm}$ and $7 \times 10^{-28} \mathrm{C} \cdot \mathrm{m}=3 e \cdot \mathrm{nm}$, respectively. Theoretical prediction ${ }^{6}$ and our numerical results agree quantitatively. Therefore, our observations allow to determine the magnitude of the dipole moment of the $\mathrm{InGaN} / \mathrm{GaN}$ QDs in growth direction. 
The identical geometry of all $\chi$-patterns lets us conclude that we observe the influence of either only one type of charge carriers or of the motion of positive and negative charges in exactly opposite directions. Remarkable is the slow time scale of the emission line shifts, which does not comply with diffusion-time constants of hot carriers. From experimental data and fit with Eq. (2), we obtain a carrier velocity on the order of $\sim 20 \mathrm{~nm} / \mathrm{s}$. It could be a weakly bound charge, propagating slowly along a threading dislocation line. Another driving force for the moving charge may be the excitation mechanism in CL: excess carriers are injected into the sample resulting in a vertical gradient of charge carriers.

In conclusion, we report the experimental proof of large dipole moments in InGaN/GaN QDs. Our results are based on the observation of characteristic traces in the spectral diffusion of the CL emission lines from single QDs. From experiment we identify built-in dipole moments as large as 0.7 to $7.0 \times 10^{-28} \mathrm{C} \cdot \mathrm{m}$. Realistic calculations using eight-band $\mathbf{k} \cdot \mathbf{p}$ theory and the Hartree model yield dipole moments of similar values. A recurrent energy-shift pattern in the luminescence of single InGaN/GaN QDs is caused by the interaction of the excitonic dipole moment of the QD and a gradually changing electric field. We propose as explanation the interaction of the QD and the changing field of a moving charge, propagating on a straight line through the material.

This work was supported by Deutsche Forschungsgemeinschaft in the frame of SFB 787.

${ }^{1}$ S. Nakamura and G. Fasol, The Blue Laser Diode (Springer, New York, 1997).

${ }^{2}$ S. Kako, C. Santori, K. Hoshino, S. Götzinger, Y. Yamamoto, and Y. Arakawa, Nature Mater. 5, 887 (2006).

${ }^{3}$ C. Kindel, S. Kako, T. Kawano, H. Oishi, Y. Arakawa, G. Hönig, M.
Winkelnkemper, A. Schliwa, A. Hoffmann, and D. Bimberg, Phys. Rev. B 81, 241309(R) (2010).

${ }^{4}$ O. Ambacher, J. Phys. D 31, 2653 (1998).

${ }^{5}$ T. Saito and Y. Arakawa, Physica E (Amsterdam) 15, 169 (2002).

${ }^{6}$ M. Winkelnkemper, A. Schliwa, and D. Bimberg, Phys. Rev. B 74, 155322 (2006).

${ }^{7}$ M. Winkelnkemper, R. Seguin, S. Rodt, A. Schliwa, L. Reißmann, A. Strittmatter, A. Hoffmann, and D. Bimberg, J. Appl. Phys. 101, 113708 (2007).

${ }^{8}$ D. P. Williams, A. D. Andreev, and E. P. O'Reilly, Superlattices Microstruct. 36, 791 (2004).

${ }^{9}$ J. H. Rice, J. W. Robinson, A. Jarjour, R. A. Taylor, R. A. Oliver, G. A. D. Briggs, M. J. Kappers, and C. J. Humphreys, Appl. Phys. Lett. 84, 4110 (2004).

${ }^{10}$ J. W. Robinson, J. H. Rice, K. H. Lee, J. H. Na, R. A. Taylor, D. G. Hasko, R. A. Oliver, M. J. Kappers, C. J. Humphreys, and G. A. D. Briggs, Appl. Phys. Lett. 86, 213103 (2005).

${ }^{11}$ R. Bardoux, T. Guillet, P. Lefebvre, T. Taliercio, T. Bretagnon, S. Rousset, B. Gil, and F. Semond, Phys. Rev. B 74, 195319 (2006).

${ }^{12}$ M. Abbarchi, F. Troiani, C. Mastrandrea, G. Goldoni, T. Kuroda, T. Mano, K. Sakoda, N. Koguchi, S. Sanguinetti, A. Vinattieri, and M. Gurioli, Appl. Phys. Lett. 93, 162101 (2008).

${ }^{13}$ V. Türck, S. Rodt, O. Stier, R. Heitz, R. Engelhardt, U. W. Pohl, D. Bimberg, and R. Steingrüber, Phys. Rev. B 61, 9944 (2000).

${ }^{14}$ J. Seufert, R. Weignand, G. Bacher, T. Kümmell, A. Forchel, K. Leordini, and D. Hommel, Appl. Phys. Lett. 76, 1872 (2000).

${ }^{15}$ Al. L. Efros and M. Rosen, Phys. Rev. Lett. 78, 1110 (1997).

${ }^{16}$ M. E. Pistol, P. Castrillo, D. Hessman, J. A. Prieto, and L. Samuelson, Phys. Rev. B 59, 10725 (1999).

${ }^{17}$ B. P. Zhang, Y. Q. Li, T. Yasuda, W. X. Wang, Y. Segawa, K. Edamatsu, and T. Itoh, Appl. Phys. Lett. 73, 1266 (1998).

${ }^{18}$ D. Bertram, M. C. Hanna, and A. J. Nozik, Appl. Phys. Lett. 74, 2666 (1999).

${ }^{19}$ R. Seguin, S. Rodt, A. Strittmatter, L. Reißmann, T. Bartel, A. Hoffmann, D. Bimberg, E. Hahn, and D. Gerthsen, Appl. Phys. Lett. 84, 4023 (2004).

${ }^{20}$ S. Rodt, A. Schliwa, K. Pötschke, F. Guffarth, and D. Bimberg, Phys. Rev. B 71, 155325 (2005)

${ }^{21}$ P. W. Fry, I. E. Itskevich, D. J. Mowbray, M. S. Skolnick, J. J. Finley, J. A. Barker, E. P. O'Reilly, L. R. Wilson, I. A. Larkin, P. A. Maksym, M. Hopkinson, M. Al-Khafaji, J. P. R. David, A. G. Cullis, G. Hill, and J. C. Clark, Phys. Rev. Lett. 84, 733 (2000). 